reaction within the vein that results in adhesion formation. The proximity of great vessels allows scarring between adjacent artery and vein. Laser extraction in thin-walled veins may involve removal or tearing of the venous wall, thus creating a communication with the adjacent artery.

\section{CONCLUSIONS}

Laser-assisted lead extraction has significantly improved the success and efficiency of lead removal. Arteriovenous fistula formation is a rare but potentially catastrophic complication. Success with endovascular techniques has provided an effective and less invasive management option. These cases stress the importance of judicious application of extraction guidelines, meticulous technique, and prompt diagnosis and management of these potentially catastrophic complications.

\section{References}

1. Bracke FA, van Gelder B, Meijer A. Arteriovenous fistula after injury of the left Internal mammary artery during extraction of pacemaker leads with a laser sheath. Pacing Clin Electrophysiol. 1999;2:833-4.

2. Kumins NH, Tober JC, Love CJ, Culbertson TA, Gerhardt MA, Irwin RJ, et al. Arteriovenous fistulae complicating cardiac pacemaker lead extraction: recognition, evaluation, and management. J Vasc Surg. 2000;32:1225-8.

3. Milla F, Mack CA, Girardi LN. Arteriovenous fistula after laser-assisted pacemaker lead extraction. Ann Thorac Surg. 2006;81:2304-6.

4. Lawton JS, Moon MR, Curci JA, Rubin BG, Smith TW, Gleva MJ, et al. Management of arterial injuries caused by laser extraction of indwelling venous pacemaker and defibrillator leads. Pacing Clin Electrophysiol. 2006;29:917-20.

5. O'Connor DJ, Gross J, King B, Suggs WD, Gargiulo NJ 3rd, Lipsitz EC. Endovascular management of multiple arteriovenous fistulae following failed laserassisted pacemaker lead extraction. J Vasc Surg. 2010;51:1517-20.

\title{
HeartWare continuous-flow ventricular assist device thrombosis: The Bad Oeynhausen experience
}

Nadia Aissaoui, MD, Jochen Börgermann, MD, PhD, Jan Gummert, MD, PhD, and Michiel Morshuis, MD, Bad Oeynhausen, Germany

The HeartWare (HeartWare International, Inc, Framingham, Mass), ventricular assist device (VAD) is a small, third-generation, implantable continuous-flow VAD. ${ }^{1}$ Despite this system's advantages, the rates of thrombus formation and thromboembolic events are not negligible and can reach $8 \%{ }^{1}$

Pump thrombus requires emergency treatment to restore an adequate flow and to avoid life-threatening complications. The therapeutic options are few: thrombolytic therapy, either systemic or local, ${ }^{2,3}$ with the accompanying risks of recurrence and bleeding, and surgical device exchange, which is associated with the problems typical of an urgent reoperative procedure. ${ }^{4}$

There are currently no specific recommendations concerning the management of pump thrombosis. This report

\footnotetext{
From the Heart \& Diabetes Center North Rhine-Westphalia, Bad Oeynhausen, Germany.

Disclosures: Authors have nothing to disclose with regard to commercial support. HeartWare is manufactured by HeartWare International, Inc, Framingham, Mass.

Received for publication Dec 5, 2011; accepted for publication Dec 14, 2011; available ahead of print Jan 16, 2012.

Address for reprints: Nadia Aissaoui, MD, Department of Thoracic and Cardiovascular Surgery, Heart \& Diabetes Center North Rhine-Westphalia, Georgstrasse 11, 32545 Bad Oeynhausen, Germany (E-mail: nadia.aissaoui@sat.aphp.fr).

J Thorac Cardiovasc Surg 2012;143:e37-9

$0022-5223 / \$ 36.00$

Copyright (c) 2012 by The American Association for Thoracic Surgery

doi:10.1016/j.jtcvs.2011.12.035
}

aims to summarize the Bad Oeynhausen experience with the management of HeartWare VAD thrombus.

\section{CLINICAL SUMMARY \\ Patients}

Between August 2009 and May 2011, 98 patients received HeartWare VADs (88 left VADs and 10 biventricular VADs) in Bad Oeynhausen, Germany. Six patients showed development of clinical signs of pump thrombosis associated with device malfunction. One patient had pump thrombosis develop on 2 separate occasions (within 6 months of each other) as a result of noncompliance with the anticoagulation regimen.

\section{Diagnosis of Device Thrombosis}

The diagnosis of thrombosis was most often made by indirect evidence. Clinical suspicion was raised in the cases of patients 1,2,3, and 4 because of documented subtherapeutic international normalized ratio values (patient 3 ), history of a transitory ischemic attack (patient 1 ), or icterus and dark urine on examination (patients 1, 2, 3, and 4). Pump checks revealed in all patients an increase of power consumption plus abnormally increased flow values differing from baseline. Laboratory tests always showed high lactate dehydrogenase and free hemoglobin levels. Demographic data are summarized in Table 1. 
TABLE 1. Patient characteristics and treatment

\begin{tabular}{|c|c|c|c|c|c|c|c|c|c|c|}
\hline Case & Age (y) & VAD & Sex & Treatment at event & Power demand $(\mathrm{W})$ & Flow rate (L/min) & INR & PTT (s) & Free Hb (mg/dL) & LDH (IU/L) \\
\hline 1 & 57 & LVAD & M & Heparin & 6.5 & 10 & 1 & 50 & 71 & 1040 \\
\hline 2 & 50 & LVAD & $\mathrm{F}$ & Phenprocoumon plus ASA & 7.6 & 7.8 & 4.1 & 45 & 561 & 5160 \\
\hline 3 & 50 & LVAD & M & Phenprocoumon plus ASA & 19.3 & 10 & 2.7 & 32 & 203 & 2340 \\
\hline \multirow[t]{2}{*}{$4^{*}$} & 48 & LVAD & $\mathrm{F}$ & None & 13.9 & 10 & 1.2 & 24 & 404 & 3660 \\
\hline & 48 & LVAD & $\mathrm{F}$ & Phenprocoumon plus ASA & 9.1 & 10 & 1.6 & 32 & 854 & 9600 \\
\hline 5 & 36 & RVAD & M & Heparin & 8.9 & 10 & 1.9 & 68 & 141 & 2390 \\
\hline 6 & 59 & RVAD & M & Heparin & 6.9 & 6.0 & 1.3 & 48 & 69 & 683 \\
\hline
\end{tabular}

$V A D$, Ventricular assist device; $I N R$, international normalized ratio; $P T T$, partial thromboplastin time; $H b$, hemoglobin; $L D H$, lactate dehydrogenase; $L V A D$, left ventricular assist device; ASA, aspirin [INN acetylsalicylic acid]; RVAD, right ventricular assist device. *Patient 4 had pump thrombosis develop on 2 occasions (within 6 months) as a result of noncompliance with the anticoagulation regimen. These occasions are listed in chronologic order.

The echocardiography was helpful because it showed indirect signs of pump malfunction in all patients with left VADs, such as left ventricular dilatation and intermittent opening of the aortic valve, but a left ventricular thrombus was identified in 1 patient (patient 1).

It was not possible to perform computed tomographic examinations, because the clinical status was often unstable. The diagnosis was always confirmed by the examination of the explanted devices showing thrombotic material inside the HeartWare VAD or the inflow cannula (Figure 1).

\section{Management}

Pump exchange. The pump exchange procedure was performed off pump or with cardiopulmonary bypass support through a median sternotomy. ${ }^{4}$ A small dose of heparin (only half of the usual dose) was used in case of off-pump surgery to avoid stasis during the exchange. In our center, off-pump surgery was preferred when the hemodynamic status of the patient was unstable.

Thrombolytic therapy. Tenecteplase was used in our 2 cases of thrombolytic therapy. Thrombolytic treatment was standardized and administered on the basis of body weight, with a maximum dose of 10,000 units $(50 \mathrm{mg})$ as a single intravenous bolus given in 5 to 10 seconds. ${ }^{5}$

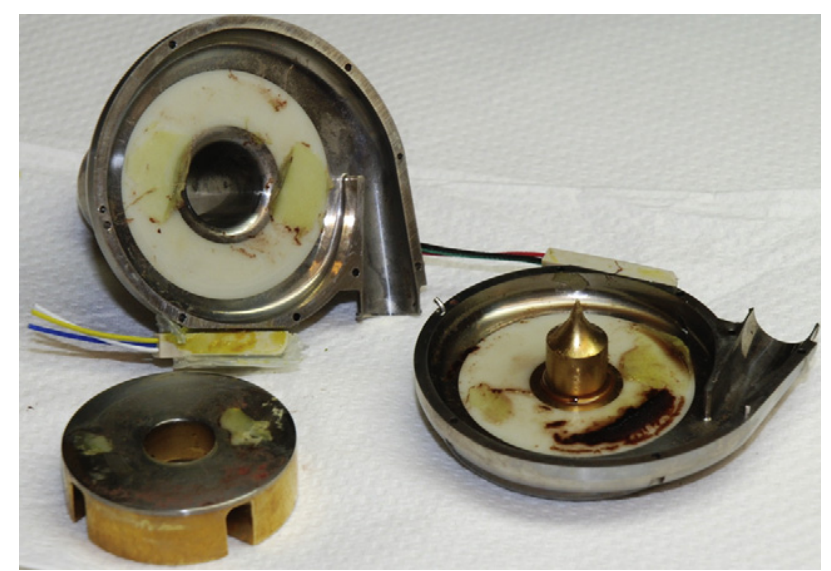

FIGURE 1. Right ventricular assist device after explantation. Fibrin and the clot can be seen inside the pump.
Because partial thromboplastin time frequently increases during lysis with recombinant tissue plasminogen activator, the heparin dose was lowered. Intravenous infusion of heparin was titrated to maintain an activated partial prothrombin time of 50 to 60 seconds, and coagulation parameters were monitored every 4 hours. After lysis, antiplatelet therapy was continued.

\section{Outcomes}

Pump exchange was successfully performed in 5 patients (patients 1, 2, 4, 5, and 6), in 3 cases with the off-pump technique (patients 1, 5, and 6). No severe bleeding or tamponade occurred in the patients treated by surgery.

Patient 3 was initially treated with thrombolytic therapy, but this failed on day 4 . Surgery was needed because there was severe bleeding at the driveline and administration of an additional dose of thrombolytic agent was not feasible.

Because of poor compliance and discontinuation of anticoagulation therapy, patient 4 had a second episode of pump thrombosis 6 months after the first. He was treated with thrombolytic therapy, which was successful and free of complications.

All patients were discharged from the intensive care unit. Patient 6 died of sepsis 1 month after pump exchange.

\section{DISCUSSION}

Pump exchange, unlike thrombolytic therapy, was always successful in our series. Thrombolytic therapy potentially induces fast thrombolysis. Tenecteplase is a thirdgeneration thrombolytic agent with a longer plasma half-life, better fibrin specificity, and higher resistance to inhibition by plasminogen activator than previous thrombolytic agents. ${ }^{5}$ In our study, thrombolytic therapy was unsuccessful in 1 of 2 patients. In the largest literature study, by Rothenburger and colleagues, ${ }^{2}$ thrombus recurred after thrombolysis 3 times in 8 patients. In fact, thrombolytic therapy cannot completely eradicate the thrombus, which may immediately or quickly rebuild after thrombolysis. Furthermore, systemic thrombolysis leads to a secondary hypercoagulable state, which may persist for 3 days. ${ }^{5}$ Finally, 
thrombolysis has adverse effects, including hemorrhage, such as occurred in patient 3 in whom thrombolysis failed.

Some authors have reported cases of successful local thrombolysis in cases of pump thrombosis. ${ }^{3}$ Such beneficial effects might be explained by high local concentrations of the thrombolytic agent. We do not think that local thrombolysis will improve the results of thrombolysis, because it still has systemic effects and is associated with bleeding complications. Moreover, the use of catheters may be dangerous in the vicinity of the continuous inflow cannula, and the examination requires the use of contrast agent and irradiation.

Pump exchange permits complete replacement of the device. No thrombus remains. Pump replacement does have the shortcomings of all reoperative procedures, ${ }^{4}$ exposing patients on antithrombotic therapy to the risk of major bleeding complications, right ventricular failure and infection. Off-pump implantation of the VAD, however, greatly shortens the duration of the procedure and may avoid the adverse effects of cardiopulmonary bypass, such as need for transfusions and systemic inflammation. ${ }^{4}$ We performed off-pump surgery in 3 cases. Despite difficulty sometimes in seeing inside the ventricle in the absence of cardiopulmonary bypass, we could always in our patients evacuate the thrombotic material in the ventricle. Successful use of offpump surgery for implanting VADs has been reported in the literature ${ }^{4}$ but requires further confirmation.

Pump exchange is an effective treatment for HeartWare VAD thrombosis. The associated risk is acceptable. Offpump surgery may reduce the risks observed with reoperative surgery.

We thank Daniela Roefe, VAD coordinator, and Ellen von Roessing for their contributions.

\section{References}

1. Strueber M, O'Driscoll G, Jansz P, Khaghani A, Levy WC, Wieselthaler GM, et al. Multicenter evaluation of an intrapericardial left ventricular assist system. J Am Coll Cardiol. 2011;57:1375-82.

2. Rothenburger M, Wilhelm MJ, Hammel D, Schmidt C, Tjan TD, Böcker D, et al. Treatment of thrombus formation associated with the MicroMed DeBakey VAD using recombinant tissue plasminogen activator. Circulation. 2002;106(12 Suppl. 1):189-92.

3. Kiernan MS, Pham DT, Denofrio D, Kapur NK. Management of HeartWare left ventricular assist device thrombosis using intracavitary thrombolytics. J Thorac Cardiovasc Surg. 2011;142:712-4.

4. Gregoric ID, La Francesca S, Myers T, Cohn W, Loyalka P, Kar B, et al. A less invasive approach to axial flow pump insertion. J Heart Lung Transplant. 2008;27:423-6.

5. Dunn CJ, Goa KL. Tenecteplase: a review of its pharmacology and therapeutic efficacy in patients with acute myocardial infarction. Am J Cardiovasc Drugs. 2001; 1:51-66. 